\title{
Thrombotic Thrombocytopenic Purpura (Syndroma Moschkowitz), Severe Disease with Potential Fatal Outcome
}

\author{
Svetlana Stankovikj ${ }^{1}$, Kata Martinova ${ }^{2}$, Vera Stankovikj ${ }^{3}$
}

\begin{abstract}
Thrombotic thrombocytopenic purpura (TTP) is a rare disease with an untreated mortality rate of 90\%. The disease consists of the pentad of microangiopatic hemolytic anemia, thrombocytopenic purpura, neurologic abnormalities, fever and renal failure. TTP, inherited or autoimmune, is mainly caused by the plasma deficiency of the von Wilebrand factor cleaving protease, ADAMTS13, owing to gene mutations or autoantibodies. The prompt delivery of plasma exchange (PEX) has reduced the mortality rate to 10-20\%. Daily $P E X$ should continue for a minimum of 2 days after platelet count has been $>150 \times 10(9) / l$. In refractory cases, the use of anti CD20 monoclonal antibody is recommended. Vincristine also has been shown to be useful in refractory patients.
\end{abstract}

Keywords: Thrombotic thrombocytopenic purpura, plasma exchange, HUS, Rituximab, microangiopatic hemolytic anemia

\section{Introduction}

Thrombotic thrombocytopenic purpura (TTP) is a rare multisystem disease characterized by massive occlusion of small blood vessels (arterioles and capillaries) by platelet rich and fibrin thrombi, resulting in a low platelet count. In its full-blown form, the disease consists of the pentad of microangiopathic hemolytic anemia (MAHA), thrombocytopenic purpura, neurologic abnormalities, fever, and renal disease (1).

TTP is a rare disease, with a reported incidence of six cases per million per year. It is an important diagnosis to make because the untreated mortality rate is $90 \%$. With plasma exchange (PEX), the mortality rate is reduced to $10-20 \%$. (2). Early death still occurs: approximately half of the deaths occurred within $24 \mathrm{~h}$ of presentation. An ethnic predisposition to TTP is not established. In the larger series reported a female predominance of approximately 2:1 has been noted.

\section{Pathogenesis}

In the last 15 years there has been a marked increase in the understanding of the pathogenesis of TTP (2). It is now recognized that congenital and acute acquired TTP are due to a deficiency of von Willebrand factor (VWF) cleaving protein, also known as ADAMTS13 metalloproteinase. In the absence of ADAMTS13, ultra large multimers of VWF released from endothelium are not cleaved appropriately, and cause spontaneous platelet aggregates in conditions of high shear, such as in the microvasculature of the brain, heart and kidneys, resulting in their dysfunction.

Congenital TTP is due to an inherited deficiency of ADAMTS13, but acquired immune TTP is due to the reduction of ADAMTS13 by autoantibodies directed against this metalloproteinase(2). The clinician must recognize the similarity between TTP and hemolytic-uremic syndrome (HUS) that can be as atypical hemolytic uremic syndrome (aHUS) and Shiga-toxin-producing Escherichia coli hemolytic uremic syndrome (STEC-HUS). Caused by infectious agents, the latter is usually distinguishable from
TTP and aHUS owing to the prodromal occurrence of bloody diarrhea after the ingestion of contaminated food or water (3). The differential diagnosis between TTP and aHUS is more difficult. While most TTP cases are due to plasma deficiency of ADAMTS13, aHUS is often caused by the abnormal activation of the alternative complement pathway.

\section{Presentation}

Patients with TTP typically report an acute or sub-acute onset of symptoms related to neurologic dysfunction, anemia, or thrombocytopenia. Neurologic manifestations include alteration in mental status, seizures, hemiplegia, paresthesias, visual disturbance, and aphasia (4). Anemia may be accompanied by fatigue. Severe bleeding from thrombocytopenia is unusual, although petechiae are common. Fever occurs in approximately $50 \%$ of patients. Patients may notice dark urine from hemoglobinuria. TTP can affect any organ system, but involvement of the peripheral blood, the central nervous system and the kidneys causes the clinical manifestations.

Clinical differentiation of HUS and TTP can be problematic. Differentiation is often based on the presence of central nervous system involvement in TTP and the more severe renal involvement in HUS.

\section{Physical}

Patients with TTP or HUS have no characteristic physical findings. Findings upon examination depend on the severity of involvement of the target organ systems. Hemolytic anemia and thrombocytopenia cause pallor, jaundice and petechiae. Abnormal findings upon neurologic examination consist of mental status changes and/or focal neurologic deficits. The defects can be evanescent and, thus, present as transient ischemic attracts. Organomegaly is not typical (5). Diagnosis

Laboratory studies for suspected TTP usually include full blood count, blood smears, coagulation studies, BUN creatinine, serum bilirubin, lactate dehydrogenase (LDH) and direct antiglobulin test (Coombs). Full blood count and blood smear will demonstrate anemia, thrombocytopenia 


\section{International Journal of Science and Research (IJSR) \\ ISSN (Online): 2319-7064 \\ Index Copernicus Value (2013): 6.14 | Impact Factor (2014): 5.611}

and fragmented red blood cells (schistocytes) (6). Clotting screen including fibrinogen is normal. Urea and electrolytes can demonstrate renal impairment, while liver function tests are usually normal. LDH and bilirubin (direct and total) levels are indirect measures of the degree of hemolysis. The level of LDH in the $1000 \mathrm{IU} / \mathrm{L}$ range (normal $<200 \mathrm{IU} / \mathrm{L}$ ) is not unusual. Generally, a moderate degree of hyperbilirubinemia is present, with the indirect form predominating. Direct antiglobulin test (Coombs) which determines the presence of antibodies on red cells is negative.

Typical cases of congenital and acquired TTP are characterized by severe ADAMTS13 deficiency in plasma (lower than 10\%) (7). The diagnosis of TTP based on very low ADAMTS13 is reinforced by finding mutations in the gene encoding the protease in the rarer congenital form or through the detection of autoantibodies against the protease in the more frequent acquired forms.

\section{Differential Diagnoses}

The clinician must recognize the similarity between TTP and HUS but in addition, the differential diagnosis also includes immune thrombocytopenic purpura (ITP) and disseminated intravascular coagulation (DIC), two entities with very different modes of therapy. In the pathogeneses of ITP, antiplatelet antibodies are the main causing factors and in DIC there is thrombin excess, in comparison with TTP, where endothelial defect can be found. Schisocytes can be seen in TTP and DIC but not in ITP. Prothrombin time (PT) and partial thromboplastine time (PTT) are normal in ITP and TTP but they are increased in DIC. Fibrinogen is normal in ITP and TTP but it is decreased in DIC. Fibrin degradation and D-dimers are normal in ITP, slightly increased in TTP and increased in DIC.

\section{Management}

The therapy of choice for TTP is plasma exchange with fresh frozen plasma (FFP). Only the minority of TTP patients $(20-30 \%)$ present with the classic pentad. The presence of microangiopathic hemolytic anemia (schistocytes, elevated LDH and indirect hyperbilirubinemia) and thrombocytopenia in the absence of other obvious causes (DIC, malignant hypertension) is justification to begin total plasma exchange (PEX). Daily PEX, preferably with spun apheresis, is the mainstay of treatment and has reduced mortality rates, from over $90 \%$ to $10-20 \%(8)$. It allows removal of autoantibody, and repletes ADAMTS13. The duration of PEX and the number of procedures required to achieve remission is highly variable. In patients refractory to plasma exchange, using cryopoor plasma (cryosupernatant) can sometimes lead to a response. This is FFP that has had the cryoprecipitate removed and is thus depleted of high-molecular weight von Willebrandmultimers, which have a pathogenic role in TTP (9). Daily PEX should continue for a minimum of 2 days after platelet count has been $>150 \times 10(9) / 1$ and then stopped. Corticosteroids may also be used in refractory patients (methylprednisolone i.v. 1g/day for 3 days or oral prednisolone $1 \mathrm{mg} / \mathrm{kg} /$ day). Oral folic acid $5 \mathrm{mg} /$ day is advisable.
Increasing evidence supports the use of the anti-CD20 monoclonal antibody Rituximab in cases of TTP refractory to plasma exchange (10). British guidelines recommended offering Rituximab to patients with refractory or relapsing immune-mediated TTP, and considering Rituximab as part of first-line therapy, along with PEX and steroids, in acute idiopathic TTP with neurological/cardiac pathology, as those cases are associated with high mortality. Vincristine, a vinca alkaloid, also has been shown to be useful in refractory patients. Platelet transfusions should be avoided unless lifethreatening (usually CNS) bleeding is present.

Complete responses criteria differ depending on the investigator, but they generally include the following: resolution of neurologic symptoms, normalization of hemoglobin, platelet count, LDH and bilirubin and normalization of creatinine. Shumak et al reported that more than one third of patients who survive an acute episode of TTP will have at least one relapse in the following 10 years. French researchers found that patients who have had acute TTP are at increased risk for development of an autoimmune disorder - most often, systemic lupus erythematous (SLE) or Sjogren syndrome, for as long as 12 years afterwards (11).

\section{Conclusion}

Thrombotic thrombocytopenic purpura is a severe disease with an untreated mortality of $90 \%$. Typical cases of TTP are characterized by severe ADAMTS13 deficiency in plasma, lower than $10 \%$. In suspected TTP patients with the presence of microangiopathic hemolytic anemia (schistocytes, elevated LDH and indirect hyperbiliribinemia) and thrombocytopenia in the absence of obvious causes, treatment with total plasma exchange should be started immediately, preferably within 4-8 hours. With this prompt treatment, the overall response rate has come up to $75-90 \%$ with the early mortality rate of $10-20 \%$.

\section{References}

[1] Thrombotic Thrombocytopenic Purpura,Theodore Wun, MD, FACP; Chief Editor: Srikanth Nagalla, MBBS, MS, FACP more.. Medscape, Nov 18, 2015.

[2] [Guideline] Scully M, Hunt BJ, Benjamin S, Liesner R, Rose P, Peyvandi F, et al. Guidelines on the diagnosis and management of thrombotic thrombocytopenic purpura and other thrombotic microangiopathies. $\mathrm{Br} J$ Haematol. 2012 Aug. 158 (3):323-35. [Medline].

[3] Bianchi, V., Robles, R., Alberio, L., Furlan, M. \&Lammle, B. (2002) Von Willebrand factor-cleave-in protease (ADAMTS13) in thrombocytopenic disorders: a severely deficient activity is specific for thrombotic thrombocytopenic purpura. Blood, 100, 710-713.

[4] Lau DH, Wun T. Early manifestation of thrombotic thrombocytopenic purpura. Am J Med. 1993 Nov. 95(5):544-5. [Medline].

[5] Tsai HM. Thrombotic thrombocytopenic purpura and the atypical hemolytic uremic syndrome: an update. Hematol Oncol Clin North Am. 2013 Jun. 27(3):565-84. [Medline].

[6] The complex differential diagnosis between thrombotic thrombocytopenic purpura and the atypical hemolytic uremic syndrome: Laboratory weapons and their impact

\section{Volume 5 Issue 2, February 2016}




\section{International Journal of Science and Research (IJSR) \\ ISSN (Online): 2319-7064}

Index Copernicus Value (2013): 6.14 | Impact Factor (2014): 5.611

on treatment choice and monitoring.Mannucci PM1, Cugno M2.Thromb Res. 2015 Nov;136(5):851-4.

[7] M. Furlan, R. Robles, M. Galbusera, G. Remuzzi, P.A. Kyrle, B. Brenner, et al., von Willebrand factorcleaving protease in thrombotic thrombocytopenic purpura andthe hemolytic-uremic syndrome, N. Engl. J. Med. 339 (1998) 1578-1584.

[8] Bell WR, Braine HG, Ness PM, Kickler TS. Improved survival in thrombotic thrombocytopenic purpurahemolytic uremic syndrome. Clinical experience in 108 patients. N Engl J Med. 1991 Aug 8. 325(6):398-403. [Medline].

[9] Marn Pernat A, Buturovic-Ponikvar J, Kovac J, et al. Membrane plasma exchange for the treatment of thrombotic thrombocytopenic purpura. Ther Apher Dial. 2009 Aug. 13(4):318-21. [Medline].

[10]Becerra E, Scully MA, Leandro MJ, Heelas EO, Westwood JP, De La Torre I, et al. Effect of rituximab on B-cell phenotype and serum B-cell activating factor levels in patients with Thrombotic Thrombocytopenic Purpura. Clin Exp Immunol. 2014 Oct 22. [Medline].

[11] Scully M, McDonald V, Cavenagh J, et al. A phase 2 study of the safety and efficacy of rituximab with plasma exchange in acute acquired thrombotic thrombocytopenic purpura. Blood. 2011 Aug 18. 118(7):1746-53. [Medline]. 